\title{
Malnutrición fetal
}

DRA. MAFALDA RIZZARDINI P,*

Fundamentalmente se distinguen tres categorías de recién nacidos con déficit significativo de crecimiento intrauterino: los que han sido concebidos genéticamente pequeños ya sea porque los padres son de talla muy baja o porque tienen malformaciones congénitas asociadas a enanismo (1, $2,3)$; los que han padecido de infecciones crónicas durante el embarazo (2), capaces de limitar el crecimiento fetal y los que no se desarrollan normalmente por insuficiente nutrición intratuterina. Estos últimos son Ios que propiamente se Ilaman malnutridos fetales.

\section{Etiología y factores asociados}

Numerosos factores pueden retardar el crecimiento del feto "in útero" actuando directa o indirectamente sobre la suficiencia placentaria o las líneas de abastecimiento materno-fetales.

En forma arbitraria podríamos dividir los factores etiológicos en materno-placentarios y ambientales sin desconocer que pueden existir otros aún no definidos.

Factores materno-plocentarios. Las madres cuyas tallas están por debajo de $1,50 \mathrm{~m}$., frecuentemente tienen nifnos cuyo peso de nacimiento es inferior al esperado para la edad gestacional $(4,5$, 6), independiente de la talla del padre. Este menor desartollo del feto "in útero" parece depender más que de la talla materna, del volumen cardíaco de la embarazada porque las mujeres de talla muy bajas, con volumen cardiaco normales ( 385 a 455 $\mathrm{ml} / \mathrm{m}^{2}$ de superficic corporal) tienen niños de tamaño nomal (7). En cambio mujeres relativamente altas con volumen cardíaco insuficiente por enfermedades cárdiovasculares, también dan a luz

\footnotetext{
* Departamento de Pediatría. Hospilal R. del Río. Santiago Chile.
}

niños subdesarrollados. Lo mismo ocurre con las madres que padecen de hipertensión u otros trastornos vasculares que limitan la circulación placentaria, como ocurre en la toxemia gravídica (2, $4,6,8,9)$. Estos hechos demuestran que el factor más importante en el retardo de crecimiento intrauterina es la disminución del flujo sanguíneo al feto $(10,11)$. El mismo efecto limitante tendría el sangramiento materno en la últimas cuatro semanas $(2,9)$, la patología del cordón umbilical como nudos verdaderos, las grandes alturas (12) y el tabaquismo exagerado de la madre $(13,14)$.

En animales se ha demostrado que ligando la arteria uterina de un cuerpo el feto de ese cuerno tiene un peso correspondiente al $65 \%$ de lo esperado (15). En corderos gemelos se ha visto que la ligadura de una arteria umbilical limita considerablemente el desarrollo del feto afectado mientras que su hermano se desarrolla normalmente (16). En estos casos se produce atrofia de la vena satélite y aumento de la presión en la placenta lo que origina importantes cambios vasculares.

Está suficientementc documentado que existe una estrecha correlación entre el peso seco y fresco de la placenta y el peso del niño al nacer. Falkner (17), en 41 pares de gemelos ha demostrado que esta relación es bastante exacta y que un niño grande generalmente tiene una placenta grande al mismo tiempo que uno pequeño tiene una masa placentaria proporcional a su peso. Hacen excepción los pocos casos de transfusión placentaria donde ambas masas pueden ser iguales, pero la irrigación es diferente debido a las anastomosis arteria-vena y probablemente arteria-arteria o vena-vena.

En gemelos discordantes se ha visto que aunque tienen una sola masa placentaria ésta está irregularmente dividida correspondiendo a cada 
niño nua parte proporcional a su peso de nacimiento (17a). Sin embargo Beargie (10) en 45 recién nacidos con grave déficit de crecimiento intrauterino observa que cl peso de la placenta tiene un cuociente de $4,6 \mathrm{cll}$ vez de 5,6 que es lo nornal. Esto significa que ella, aunque pequeña para la edad, cs grande para el niño.

El peso de la placenta aumenta en forma lineal durante toda la gestación, siendo al término del embarazo $1 / 5$ a $1 / 6$ del peso del niño (18). En la figura 1 se señala el peso de las placentas correspondientes a recién nacidos a término cuyo peso estaba por debajo del percentil 10 para la edad. Se ve que los pesos de las placentas están por debajo del percentil 50 excepto en 4 casos. Uno cortesponde a una placentá única de dos gemelos univitelinos, que se pesó en conjunto y otro a un niño mongólico cuya placenta se sabe que es de tamaño normal, ya que la causa de su déficit de crecimiento es genética.

E] crecimiento de la placenta es un proceso continuo de replicación celular. Según Winick (19), el DNA de la placenta aumenta en forma lineal hasta que pesa 350 gramos y el feto $2.300 \mathrm{~g}$. Luego se produce una desaceleración gradual de esta multiplicación celular. Filer (20) comunica algo parecido, pero encuentra que cuando las condiciones ambientales son más favorables, el crecimiento lineal llega hasta $450 \mathrm{~g}$. En gemelos el DNA de las placentas es muy proporcional al peso del niño y al peso de las placentas secas o frescas por lo que el simple peso de este órgano, excepto contadas excepciones, es un indice de crecimiento real de clla y no parece necesario hacer estudios bioquímicos para estimarlo con mayor seguridad (21).

El mismo Winick en 1967 (22) comunicó que las placentas de los niños que padecen de déficit de crecimiento intrauterino tienen un menor número de células, de un tamaño significativamente más pequeño (contenido de DNA y RNA menor). Otros autores han encontrado que la mayor parte de estos niños tienen anomalías de irrigación desde los vasos uterinos al cordón (11), infartos o zonas avasculares y menor volumen total de las vellosidades (23) Shanklin (24) estudiando 6.500 placentas indica que sólo cuatro condiciones tienen un posible papel er la patogenia del peso subnormal al nacer: inserción anormal del cordón, transfusión parabiótica en gemelos monocoriales, fibrinosis difusa y hemangiomas de tipo sinusal. Estas tres últimas son situaciones bastante raras, to así las inserciones anormales del cordón que correspondieron al $1,51 \%$ de todos los embarizos $y$ al $6,9 \%$ de las gestaciones múltiples.

El problema en todo caso es bastante complejo. No siempre se encuentran lesiones anatómicas que limiten la circulación placentaria y probablemente algún papel juega en al desirrollo del felo la dinúmica materno-feto-placentaria y un cantiderd total de nutrientes disponibles y transfcribles, independientemente del peso absoluto o de las lesiones macroscópicas de la placenta.

Factores ambientales. La asociación entre pobreza y reducción del peso del recién nacido es bien conocida pero aparentemente ello se debc a que entre Ias clases sociales menos favorecidas son más frecuentes la toxemia, la primiparidad precoz, la gran multiparidad, condiciones todas que limitan el buen desarrollo fetal.

En animales se ha demostrado que la malnutrición de la madre al final de la gestación produce crías de menor tamaño que lo esperado (14). Dobbing (25) en ratas y mediante estudios bioquímicos postula que la desnutrición materna aunque sea moderada influye en el desarrollo corporal y del cerebro y hay reducción de la mielinización medida por la concentración de colesterol.

Teóricamente, la malnutrición fetal en la especic humana podría ser la consecuencia de la desnutrición materna o de un menor contenido de algunos nutrientes en la sangre de la madre pero esto no se ha demostrado en forma fehaciente. E! feto humano estaría mucho más protegido que el de los animales de los efectos deletéreos de la desnutrición materna. Gruenwald en 1967 (26), observó que cuando en Japón, las condiciones sociocconómicas mejoraron sustancialmente, después de 20 años de pobreza secundaria a la guerra, el peso del recién nacido no aumentó significativamente. A pesar de la relativa prosperidad actual del Japón el peso del recién nacido es sólo 200 gramos mayor. En promedio menos del 10\%. Lo mismo ocurre cuando hay una escasez aguda de alimentos como en Rotterdam, durante la ocupación alemana (27). Las madres en vez de subir 12 kilos durante el embarazo, subían sólo 2 kilos pero el peso de nacimiento en promedio sólo sc redujo en un $7 \%$. En una y otra experiencia no hay información sobre el porcentaje de nacimientos prematuros y otros factores como cambios en la estructura de la población, de la tensión psicológica y del riesgo de infección, factores todos quc pueden haber influido en el peso promedio de los recién nacidos.

Parece que la desnutrición severa y aguda de la madre condiciona más bien infertilidad, aumento del número de abortos que reducción del tamaño del feto.

Cuando se trabaja con encuestas alimentarias los resultados obtenidos son aún de más difícil interpretación. Muy conocidos son: el estudio en un grupo de mendigos (28) en el que no se encentró diferencias en el peso de sus recién nacidos comparado con la población gencral y el dc Mac Ganity en USA (29), que incluye 2.046 madres y el que no reveló asociación alguna entre la ingesta materna y cl peso del recién nacido. 
Por último cuando se da un suplemento calórico a la madre crónicamcnte desnutrida durante el embarazo, parece que el peso del recién nacido aumenta en forma significativa (30), Lechtig (31) y el grupo del INCAP han demostrado tambjén que la Incaparina con leche usadas como suplementación alimentéria durante la gestación mejora la velocidad del crecimiento fetal. Desafortunadamente estits experiencias con suplementación alimentaria en gestantes, no han dado siempre los mismos resultados. Otros investigadores no han logrado demostrar un efecto definitivo sobre el peso del recién nacido (32).

Walker (4) en 14.485 partos a término estudiados encuentra que en el $59 \%$ de los casos de malnutrición fetal no se logra encontrar una causa que justifique el menor peso del niño. En el $15 \%$ hay antecedentes de toxemia o hipertensión de otro origen y en el $4,9 \%$, sangramientos anteparto. Beargie (10) en 45 niños encuentra que casi la mitad no tienc antecedentes maternos o de complicaciones durante el embarazo. En cambio, Gruemwaldru (33) incluyendo malformados, informa que en el $79 \%$ de los casos hay complicaciones. (Tabla 1). Estos hallazgos no descartan la posibilidad que haya algún factor materno asociado a déficit de crecimiento intrauterino, ya que cn todas las casuísticas disponibles se reconoce el carácter repetitivo de esta condición $(13,34,35)$. Algunos autores informan que el $25 \%$ de las madres estudiadas han tenido otro niño malnutrido fetal y el $10 \%$ varios niños semejantes $(4,13)$.

TABLA NO 1

YT?

CONDICIONES ASOCIADAS A RETARDO DEL CRECICIMIENTO EN 63 CASOS. SANTIAGO.CHILE

\begin{tabular}{|c|c|c|}
\hline & $N^{o}$ & $\%$ \\
\hline $\begin{array}{lllllllll}\text { Gemelos } & \ldots & \ldots & \ldots & \ldots & \ldots & \ldots & \ldots & \ldots\end{array}$ & 6 & 9,52 \\
\hline Toxemia ....... .. . . . . & 10 & 15,87 \\
\hline 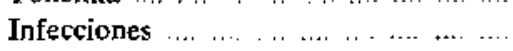 & 4 & 6,34 \\
\hline \multicolumn{3}{|l|}{ Connatales } \\
\hline 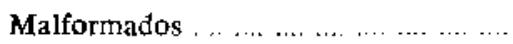 & 9 & 14,29 \\
\hline Patología del cordón $\ldots \ldots \ldots \ldots \ldots \ldots$ & 1 & 1,59 \\
\hline de la placenta $\ldots . . . . . . . . . .$. & 12 & 10,05 \\
\hline Sin antecedentes ....... & 34 & 53,96 \\
\hline
\end{tabular}

\section{Incidencia}

La mayor parte de los investigadores han comunicado que aproximadamente el 30 al $35 \%$ de los recién nacidos de peso inferior a 2.500 gramos han nacido a término $(3,6,36)$. Beargie $(12)$ en Kentucky, en un medio socio-económico relativamente bajo, en el que hay muchos negros encuentra que el $4,5 \%$ de los nacidos vivos, son a término pequeños para la edad (cl porcentaje total de niños de bajo peso es de 13,5\%). Lubchenco y Battaglia (37) observan que pot lo menos el $10 \%$ de todos los nacimientos corresponden a niños subdesarrollados y Yerushadmy (38) analizando el mismo material de una incidencia de $5,7 \%$ en la raza blanca y $11,1 \%$ en la negra. Walker (4) en Dundce encuentra que el $1,5 \%$ de todos los nacimientos y el $24 \%$ de los de bajo peso son pequeños para la edad y Gruenwald (39) que el $3 \%$ de los niños que nacen vivos y el $24 \%$ de los que mueren en el período perinatal tienen al nacer un peso menor que el esperado para su tiempo de gestación.

Por otro lado Scott y Usher (35) tomando en cuenta el aspecto físico en 3.714 recién nacidos observa que el $10 \%$ tienen evidencia de malnutrición sin que influya sobre este porcentaje la talla materna o el sexo.

Diagnóstico. En algunas madres que han dado a luz niños con retardo de crecimiento se ha observado que existe una concentración crónicamente baja de estriol en la orina (15). Hay, sin embargo, dificultades para establecer cifras absolutas normales de estriol urinario para una edad gestacional dada. Beiscker (40) en 567 embarazadas encuentra que cuando la excreción de estriol es normal, cl peso promedio del recién nacido después de 36 semanas es $\mathbf{3 . 4 4 1}$ g. Cuando las cifras de estriol están en el límite más bajo, el promedio es sólo de $3.134 \mathrm{~g}$, diferencias que son estadísticamente significativas. El peso placentario en ambos grupos también evidenció francas diferencias.

Según Walker (4) el estriol urinario tiende a disminuir a las 32 semanas de gestación en el retardo de crecimiento intrauterino, pero el utilizar la excreción de estriol como auxiliar para el diagnóstico de la malnutrición fetal puede tener valor sólo cuando se sospecha que el niño está en riesgo $y$ es necesario extraerlo porque hay otras situaciones en las que existe una menor excreción de estas substancias extrogénicas, que nada tienen que ver con déficit de crecimiento intrauterino. EI análisis cuantitativo es difícil, hay fluctuaciones amplias de un día a otro y en la misma paciente $y$ el procedimiento enpleado no siempre es exacto y específico.

Hay numerosas publicaciones que señalan la utilidad de estudiar el líquido amniótico para precisar edad fetal y por lo tanto, grado de crecimiento, pero los datos son insuficientes lo que impide su uso indiscriminado. La edad menstrual y obstétrica estimada en forma cuidadosa pucde dar mayores rendimientos.

El reconocimiento del malnutrido fetal en et momento del nacimiento no es difícil cuando presenta signos de malnutrición. En algunos cnsos, sin embargo estos signos no existen en cl momento 


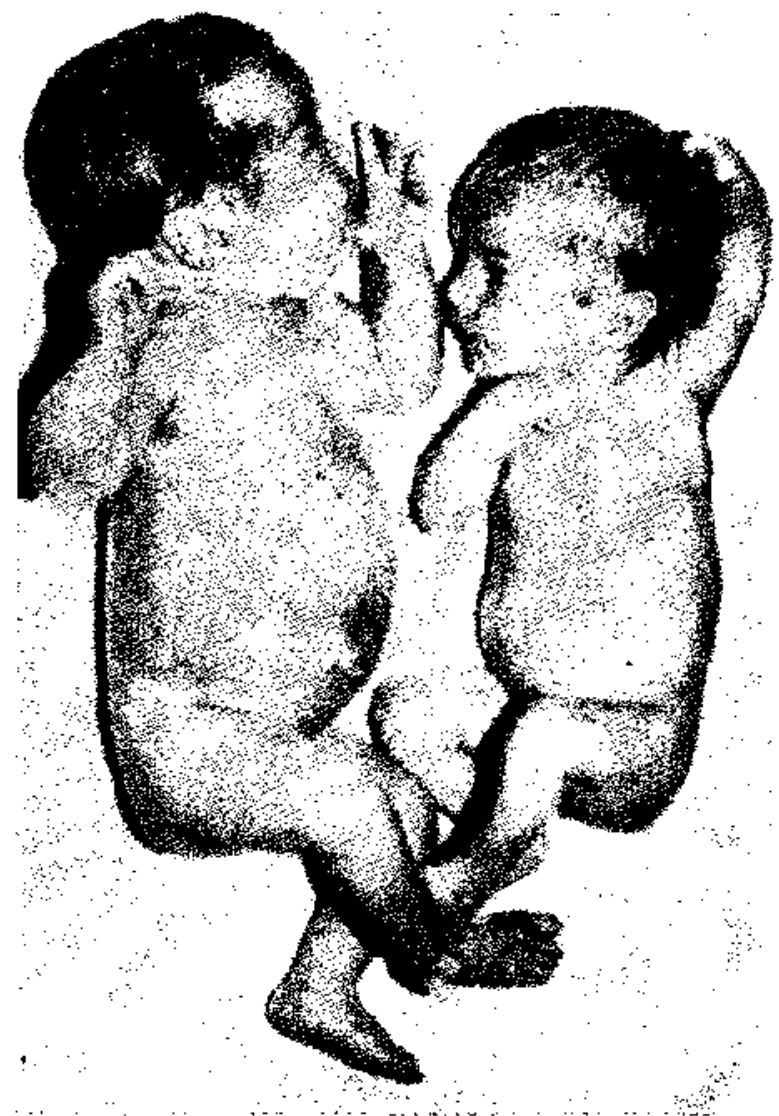

Fig. 2.- Majnutrido inlrauterino, hijo de madre toxemica de 40 semanas de gestación y $1.820^{\circ}$ gamos cle peso. A la jzquierda un recien jiacido de la misma edad gesiacional con peso de macimiento normal 3.420 grs.

del nacimiento, pero pasadas las primeras horas de vida se advierte que el niño al perder el turgor propio del recién nacido, se ve enflaquecido, parece deshidratado sin presentar pliegues positivos ni fontanela deprimida, los surcos subparpebrales son muy marcados, permanecen con los ojos abiertos, desarrollan gran actividad, rara vez necesitan incubadora pasadas las primeras horas de vida, se alimentan con avidez, toleran bien grandes volítmenes de leche los que succionan directamente de la mamadera cosá que no ocurre con los prematuros verdaderos del mismo peso (Fig. 2). El examen físico demuestra que los signos de madurez están todos presentes. El pelo es abundante, las orejas bien formadas con cartílagos firmes, hay abundantes y profundos pliegues plantares. El nódulo mamario puede estar reducido al mínimo pero siempre está presente cuando el niño es a término a cerca de él por muy comprometido que esté el estado nutritivo (41). El examen neurológico $(42,43)$ y el electroencefalograma $(44) \mathrm{com}$ rresponde también a su grado de inadurez aunque en algunos casos hemros visto que las respuestas no son adecuadas a su edad gestacional (41) como ocurre en el recién nacido bien desarrollado.
Hallazgos semejantes han sido comunicados por otros autores $(45,46)$ quienes encuentran que hay una menor integración espinal y supraespidal del control motor de las neuronas en los malnutridos intrauterinos lo que es un signo más del retardo del crecimiento.

Reinterrogada li madre, si no se Iogra establecer la fecha exacta de la última menstruación frecuentemente se observa que el nir̆o ha crecido menos de lo esperado "in utero". No es raro que la misma madre ha advertido que el vientre ha crecido menos en este embarazo que en otros y que incluso el profesional que la tha examinado ha puesto en duda la duración de la gestación, basándose sólo en la altura uterina o en la palpación de los segmentos fetales.

Aspectos clínicos. Hemos descrito el aspecto extcrior del malnutrido al referìnos al diagnóstico y hemos señalado que no todos los recién nacidos con déficit de crecimiento intrauterino presentan signos clínicos de desnutrición aunque pueden tener muy disminuidos sus depósitos de grasa subcutánea y especialmente de grasa negra. En tanto si aparecen emaciados o no al nacer se puede advertir que el cordón umbilical es may delgado, a menudo amarillento o impregnado de meconio y que rápidamente se endurece y deseca.

Al colocar el peso, la talla, el perímetro cefálico y la proporción peso/talla en las curvàs de crecimiento normales y compararlas con las de los prematuros se puede observar que tanto el peso como la talia y el perímetro cefálico están seriamente comprometidos aunque el peso es la medida antropométrica más deleprimida. En el material de Usher (11) los niños que presentan problemas tienen un peso por debajo del percentil 3 lo que según la curva de este autor (17) equivale al percentil 10 de las de Denver (47a).

Algunos autores postulan que según estas medidas podrían distinguirse tres grados de malnutrición fetal. El grado más leve sería aquel en qué sólo está comprometido el peso siendo la talla y el perímetro cefálico normales, y el ejemplo típico sería cl postmađuro. Es un niño de talla y perímetro cefálico normal pero de muy poco peso para su talla. Se ve enflaquecido, el cordón y la piel impregnada de meconio, deshidratados y con la piel desprendida en colgajos. Corresponde al dismaduro descrito por Clifford (48). El grado dos correspondería a los niños en los que está comprometido el peso y la talla pero en menor medida al perímetro cefálico. El ejemplo típico es el hijo de madre toxémica. Clínicamente es difícil distinguirlos de los prematuros verdaderos ya que la mayoría de ellos no presentan signos evidentes de desnutrición en el primer día de vida y tampoco se ven deshidratados. Sólo el comportamiento ante la alimentación, la gran actividad que de- 
sarrollan, las características físicas y neurológicas que indican madurez perniten hacer cl diagnóstico. A este grupo pertenece también el gemelo discordante, con peso inferior en un $25 \%$ al de su hermano más grande. Aunque el peso promedio de los gemelos es generalmente menor que $\mathrm{cl}$ de los embarazos únicos, sólo está expuesto il sufrir la morbilidial habitual del maluutrido fetial el más pequeño del par, cuando su peso es significativamente menor.

En muchos de estos casos además de la desigual distribución de la masa placentaria hay cortocircuitos vasculares que benefician al mejor dssarrollado.

La forma más severa de malnutrición fetal es aquella en que están tán comprometidos el peso, como el perímetro cefálico y la talla. El ejemplo más típico es el malnutrido pretérmino en el que la deficiencia de creecimiento se ha iniciado antes de 24 semanas de gestación. Al riesgo da 1a inmadurez estructural y bioquímica se agrega el riesgo adicional de la malnutrición fetal concomitante. Es excepcional en edades gestacionales muy bajas porque se necesita del factor tiempo para que las limitaciones nutricionales tengan real efecto sobre el desarrollo fetal.

Winick (49) propone una clasificación del malnutrido intrauterino basado en observaciones en animales las que extrapola al ser humano. Básicamente habría dos tipos de malnutrición fatal: uno "simétrico" en el que estarían igualmente comprometidos el peso, la talla y el perímetro cefálico del recién nacido. Experimentalmente se reproduce subalimentando a la madre desde el comienzo de la preñez y en el ser humano se asocia a subnutrición crónica. Y otra forma "asimétrica" en quc el peso estaría muy comprometido, menos la talla y casi nada el perímetro cefálico. Esta forma asimétrica se obtiene en animales de experimentación mediante ligaduras vasculares y en el ser humano corresponde a la forma clínica observada en hijos de hipertensa o toxémicas. La forma simétrica tendría peor pronóstico en lo que a desarrollo se refiere. En la forma asimétrica podría esperarse un desarrollo post-natal normal ya que el cerebro está menos comprometido.

Datos DE LABORATORlo. Las investigaciones de laboratorio del malnutrido fetal han demostrado que su homeostasis es diferente, a la del racido a término de peso normal como al pretếrmino de su mismo peso. La glicemia es mucho más baja a cualquier edad dentro de los primeros cinco días, que la de los niños de la misma edad gestacional de peso normal (50). La glicemia promedio comparada con las del prematuro del mismo peso es también relativamente más baja y por lo que están más expuestos a padecer de hipoglicemia (51).
PESO DE 50 PLACENTAS DE RECIEN NACIDOS A TERMLNO PEQUENOSOS PARA EDAD GESTACIO. NAL COMPARADOS CON VALORES PROMEDIOS PARA CADA EDAD

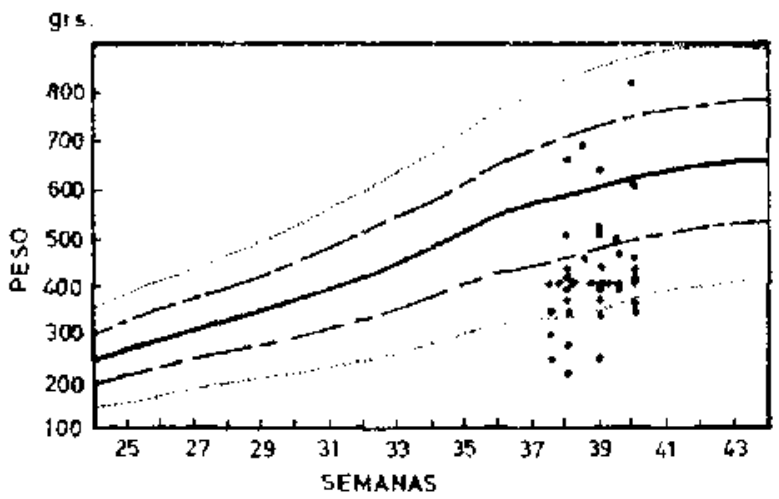

Shelley y Neligan (52) han demostrado que la hipoglicemia del malnutrido fetal es secundaria al agotamiento de los depósitos de glicógeno los que son insuficientes para suplir las necesidades durante los primeros días de vida. La hipoglicemia se asocia a aumeento de los niveles plasmáticos de ácidos grasos y cuerpos cetónicos lo que detmuestra que el malnutrido intrauterino, a falta de glicógeno, utiliza las grasas con fines energéticos $(53,54)$. Estos niños no sólo utilizan las grasas como fuente de energia sino que también las proteínas endógenas ya que los niveles sanguineos de nitrógeno, ácido úrico y urea están también significativamenete elevados (55).

La bilirrubinemia es en nuestra experiencia mucha más elevada que la de los niños normales (51) y muy semejante a la de los prematuros verdaderos. Nuestros resultados son diferentes a los comunicados por otros autores, que encuentran en los malnutridos nacidos a término diferencias significativas en los valores de bilirrubina si se les compara con los pretérmino del mismo peso (56). De todos modos conviene tener presente este riesgo adicional del malnutrido a térnino con el fin de prevenir la hiperbilirrubinemia a la que estarían tan expuestos como el prematuro verdadero.

La causia de esta hiperbilirrubinemia parece ser la destrucción del exceso de glóbulos rojos característico del malnutrido intrauterino y probablemente una menor disponibilidad de glucosa para la glucoronizaciôn.

En la sangre del cordon y en las primeras horas de vida, a menudo se encuentra intensa acidosis respiratoria y metabólica, las que desaparecen espontámeamente a las pocas horas, sustituyéndolas por alcalosis con $\mathrm{pH}$ sobre 7,50 U. y pCO2 bajo $25 \mathrm{~mm}$. Hg. (11).

En algunos casos no existe adecuada hiperventilación o hay problemas pulmonares y la acidosis 
no se corrige espontáneamente creando serios problemas cn el manejo de cstos niños.

El netabolismo expresado en consumo de $\mathrm{O} 2$ se relaciona, no con el peso sino con su edad gestacionat $(57,58)$. No hay diferencias entre el recién nacido a término y el malnutrido fetal de la misma edad, pero es relativamente superior al consumo de $\mathrm{O} 2$ del prematuro verdadero del misno peso (59).

Los niños pequeños para la edad tienen una capacidad limitada para conservar cl calor corporal $(58,60,61)$, por el pequeño tamaño y la escasa grasa subcutánea (62). Sin embargo, están en mejores condiciones para tener una adecuada tcrmoregulación que el niño más inmaduro del mismo peso a causa de un mejor tono flexor $y$ de un índice de producción de calor en reposo, mayor. La capacidad de sudoración es la correspondiente al recién nacido a término y está bien desarrollada la capacidad de producir calor como respuesta al frío aumentando la actividad muscular, generalmente sin escalofrios, $y$ aumentando la producción de catecolaminas (noradrenalina) que transforma la grasa parda. Cuando los depósitos de grasa son muy exiguos o se agotan porque la exposición al frío se mantiene un tiempo prolongado, se advierte un descenso grave de la temperatura corporal. En la hipoglicemia sintomática hay también poca respuesta al frío probablemente por la misma causa.

La desnutrición fetal se acompaña de cambios característicos en la composición corporal (63). Hay expansión relativa del compartimento extraccjular semejante a la observada en la desnutrición postnatal (64). Al comparar el volumen relativo $(\mathrm{ml} / \mathrm{K})$ del espacio de bromuro en niños malnutridos "in utero" con el recién nacido de peso nomal se advierten valores relativamente altos aunque el volumen absoluto o total está en razón directa con la madurez fetal. A mayor madurez. menor espacio extracelular, en relación al peso total.

El espacio extracelular es mayor micntras más comprometida está la longitud del meonato con déficit de crecimiento, lo que indica que la malnutrición es más grave y ha durado probablemente más tiempo. Los datos disponibles, especialmente los de Sinclair y Silverman (59) son sin embargo contradictorios y en todo caso existe una gran dispersión, probablemente por la mala clasificación del material en estudio y por las diferentes edades de los neonatos estudiados ya que no se sabe bien cuando estas alteraciones sc corrigen o si ellas son permanentes.

Los estudios bioquímicos de Winick (65) han demostrado que en el malnutrido hay un menor número de células, de menor tamaño lo que sugiere la posibilidad que también exista una menor cantidad de agua intracelular $\mathrm{y}$ de solutos corpo- tales totales semejante a lo descrito en la desnutrición postnatal. Para confirmar esta hipótesis habría que hacer simultáneamente estudios seriados de metabolismo, compartimientos líquidos, cuocientes DNA/nitrógeno no colágeno en los iejidos, lo que se está realizando en algunos centros de investigación. Otros exámenes han demostrado que el hematocrito y la hemoglobina de los niños malnutridos está significativamente elevados como respuesta a la hipoxia crónica intrauterina $(66,67)$. En estos niños se describe un sindrome llamado de hiperviscosidad sanguínea $(68,69,70,71)$ que sería muy scmejante al de las cardiopatias cianóticas (71). Este sindrome se caracteriza por síntomas neurológicos como temblores, irritabilidad y convulsiones; síntomas cardiopulmonares: disnea, ritmo respiratorio sobre 50 por minuto, aumento de tamaño del corazón con congestión pulmonar y hepatomegalia, y priapismo. En los casos más graves hay trombosis $\mathrm{y}$ hemorragias debidas a congulación intravascular diseminada. La mayor parte de estos pucientes tienen hemutocrito sobre $65 \%$ y presentan hipoglicemia asociada.

Estudios RADIOLógicos. La radiografía de huesos demuestra un serio retraso de maduración. Generalmente faltan los núcleos distales del fémur o son de tamaño muy pequeño (72). Li radiografía de tórax puede dar otro dato interesante: el timo tiene aspecto atrofiado y el mediastino superior es notablemente estrecho.

Anatomía patológica. Gruenwald en 2.400 nacimientos (39) sucesivos analiza el material de autopsia, propone una tabla standard para peso de nacimiento, talla y peso de órganos según edad gestacional y cncuentra que el cerebro del malnutrido intrauterino es relativamente grande, con sus circunvoluciones cerebrales más desarrolladas que lo esperado para su peso, correspondiendo más bien a su tiempo de gestación (73). La talla es también relativamente superior al peso y lo mismo ocurre con el bazo y el corazón. El timo aparece significativamente más pequeño debido a atrofia nuacro y microscópica, hecho que se ha observado también en el lactante menor desnutrido $y$ que evidencia el distress crónico (74). Latoche (74) en un estudio detallado macro y microscópico en 34 cerebros de niños con marcado retardo del crecimiento encuentra que el crecimicnto y la maduración morfológica son apropirdos para la cdad gestacional (75).

El riñón presenta casi todos los glomérulos vecinos a la cápsula bien diferenciados, los alvéolos pulmonares están desarrollados y la hematopoyesis extramedular del hígado es escasa o nula. El peso del hígado es significativamente menor que el esperado para el peso del niño y sus depósitos de glicógeno, muy exiguos. 
Morbilidad neonatal. La mayor parte de los malnutridos intrauterinos que fallscen en el período perinatal lo hacen antes de nacer, por astixia $(2,6,73)$. La mayoría de los que sobreviven tienen una morbilidad neonatal característica.

Así como el sindrome de membrana hialina y la hiperbilirrubinemia grave, susceptible de necesitar recambio se observan casi exclusivamente en el recién nacido pretérnino; la hipoglicenia sintomática, la hemorragia pulmonar y el sindrome aspirativo son más frecuentes en el malnutrido fetal (76).

La mayor parte han padecido de asfixia intrauterina (62), la que puede agravarse durante el parto. El líquido amniótico frecuentemente tienc meconio el que puede ser aspirado por el feto originando un sindrome de aspiración con o sin neumotórax adicional.

Muchos de estos niūos padecen de insuficiencia renal, poliglobulia, coagulopatías dependientes o no de la vitamina $K$, hipoglicemia y acidosis. También se ha sugerido que el malnutrido intrauterino sería más susceptible de contracr infecciones graves durante los primeros meses de vida debido a una menor transferencia intrauterina de IgG maternas (77). Los estudios a este respecto son sin embargo, contradictorios (78), independientemente del hecho reconocido que no todal la capacidad inmunitaria del niño está asociada a lgG.

Mortalidad. La mortalidad perinatal de los recién nacidos bajo el percentil 10 es 5 a 10 veces mayor que la de los niños de peso normal $(6,79,80,81,81$ a). Usher (11) comunicat una mortalidad perinatal de $18 \%$ en 26.453 nacimicntos entre los niños cuyo peso está bajo el percentil tres. La desnutrición fetal origina por sí sólo, según este autor, el $1,4 \%$ de todas las muertes perinatales. Walker (4) cstina en $10 \%$ la mortalidad perinatal de este grupo correspondiendo la mayor parte de este porcentaje a mortinatalidad. La mortalidad en la primera semana de vida es, en cambio muy baja, $1,5 \%$.

Otras casuísticas dan una mortalidad perinatal de $9,5 \%$ contra $0,9 \%$ en los niños de la misma edad gestacional, pero con peso normal, nacidos a término (82).

La mayor parte de las muertes perinatales ocurren "in útero", especialnente cntre las 32 y 36 semanas. Las muertes neonatales se deben a asfixia, insuficiencia cardíaci, neumotórax secundario, a aspiración masiva, hipoglicemia y hemorragias. La edad proncdio de muerte cs de 46 horas ( 8 a 114 horas). Según cste mismo autor (82) mientras más grave es el déficit de crecimiento mayor es el índice letal. Si el déficit es del 35\% muere uno de cada scis niños. Si es de $40 \%$, la mortalidad excede el $40 \%$.
Manejo en el período neonatal. Cuando se sospecha el nacimiento de un niño subulesarroIlado deben arbitrarse todos los medios para evitar que a la asfixia crónica intrauterina se agregue la del parto, la secundaria a analgésicos y a procedimientos de reanimación poco eficientes.

En vista de la escasa capacidad de estos niños para conservar til calor, deben protegerse contra la injuria del frío colocándolos en ambiente térmico neutral, durante la primera semana de vida. Así se facilita lá recuperación de lá asfixia y se evita cl agódmiento de los depósitos de glucosa y del glicógeno.

La morbilidad específica se tratará según los esquemas habituales, previniendo algunos cuadros mórbid só conto la hipoglicemia mediante la iniciación precoz de la alimentación con volúmenes de leche mayores que los tradicionales recomendados para el prematuro. Cuando no presentan patologia adicional puede permitirse la alimentacion "ad libitum".

Aunque aparentemente parezean deshidratados no se recomienda la administración parenteral de líquidos a menos que se demuestre que existe algún trastorno metabólico que haga necesario este precedimicnto. Se ha señalado que existe un espacio extracelular aumentado, que estos niños frceuentemente presental signos de insuficiencia cardíaca y a veces renal por lo que cuando es necesario administrar líquidos por vía parenteral hay que comeazar con $50-60 \mathrm{ml} / \mathrm{K} /$ día hasti asegurar la excreción renal. Los niños deben pesarse cada 12 horas y si el aumento de peso es mayor de 1 a $2 \%$ a dícl o aparece edema debe limitarse la administración de líquidos o intentai diuresis osmótica con manitol.

La acidosis del malnutrido no es necesario corregirla cuando hay una buena ventilación pulmonar. Cuando hay trastornos respiratorios se recomienda la administración de 3 a $5 \mathrm{mEq} / \mathrm{K} / \mathrm{d}$ ía de bicarbonato, en suero glucosado al $20 \%$.

El uso de los corticoides que podría tener justificación teórica no se recomienda como rutina, especialmente si se tiene en euenta la facilidad con que estos niños retienen Jíquidos con el consiguiente peligro de insuficiencia cardiaca congestiva.

La hipoglicemia es extraordinariamente frecuente en el malnutride fetal especialmente cuanJo no se inicia la alimentación muy precozmente o no se tolera bien. El diagnóstico oportuno de este trastorno metabólico sólo es posible mediante cxámenes serindos de glicemia ya que sólo aproximadamente la mitad de los niños con cifras significativamente bajas de glucosa en la sangre presentan signos sugcstivos de hipoglicemia. El tratamicnto debe ser lo más precoz posible, independientemente que haya síntomas o no, ya que no se há demostrado que la hipoglicemia neonatal 
asintomática no sea capaz de producir daño neurológico.

La alimentación, al producir una moderada hemodilución, disminuye el riesgo de hiperviscosidad pero cuando el hemotocrito sube de $65 \%$ o hay síntomas sugestivos, se recomienda hacer recambio plasmático parcial con 10 a $20 \mathrm{ml} / \mathrm{K}$. Osky (83) propone una fórmula matemática que permite bajar el hematocrito a cifras cercanas a $60 \%$, lo que la mayoría de las veces es suficiente.

Algunos alutores piensan que el tratamiento del malnutrido fetal debe comenzar "in útero" (13). No hay que permitir que un niño con déficit importante del crecimiento siga desnutriéndose cuando ha cumplido 37 semanas. Mejor es extraerlo cuidando que el riesgo de asfixia no aumente durante el parto. La reanimación debe practicarla un especialista ei que a su vez tomará en cuenta los riesgos neonatales característicos, para su mejor manejo.

Crecimiento inmediato y a largo plazo. La mayor parte de los malnutridos aumentan rápidamente de peso $y$ no presentan el descenso inicial característico del prematuro (84). Los pesos promedios colocados en las curvas de crecimiento de Dancis señalan que existe un rápido ascenso ponderal que supera con mucho a la de los prematuros verdaderos del mismo peso pero que son productos de gestaciones más cortas.

Posteriormente se observa una rápida desaceleración del crecimiento de tal modo que en la edad preescolar la mayor parte de estos niños tienen un peso, talla y perímetro cefálico bajo el percentil 10 de las curvas de Stuart $(10,84,85$, $86,87,87$ a). En cambio los prematuros verdaderos tienten tendencia a alcanzar el peso normal standard a los dos años y la talla a los cuatro años (88), aunque el peso de nacimiento sea inferior a $1.500 \mathrm{~g}$. Estudios hechos en gemelos discordantes parecen señalar también que el más liviano de cada pareja es más pequeño que su hermano a cualquier edad $(3,17,84)$.

El único observador que no encuentra diferencias en el crecimiento del malnutrido intrauterino es Pick (89) quien en tres niños severamente malnutridos al nacer observan crecimiento normal excepto en uno, hijo de padres de muy baja estatura.

En cuanto a desarrollo intelectual, Babson (3) ha observado que el QI del gemelo discordante más chico es significativamente menor que el de su hermano con buen desarrollo. Este hecho no ha logrado demostrarlo para el malnutrido fetal producto de embarazo único (90) en los que la diferencia promedio de los scores es de tres puntos, no significativo.

Beargie (10) en su reducida casuística, 45 casos, encuentra que nueve no alcanzan un desa- rrollo neurológico correspondiente a la edad cronológica, observados entre los 13 y 53 meses.

Neligan (91), a los cinco años de edad, de un QI promedio de $109,6 \%$ para los niños cuyo peso de nacimiento está bajo el percentil 5 contra $110,7 \%$ para los de peso entre los percentiles 25 y 75. Reconoce cste autor que no puede asegurarse que el QI menor del malnutrido se deba sólo a su crecimiento defectuoso ya que puede influir la hipoglicemia y la asfixin, tan frecuentes de observar en estos niños.

La casuística de Warkany (3) cs la más pesimista: $2 / 3$ de los niños con retardo del crecimiento intrauterino son, según este autor, mentalmente subnormales; sin embargo Fitzhardinge (92), en 96 niños seguidos en forma prospectiva hasta los cinco años no observa diferencias importantes en el QI, comparado con los prematuros aunque hay una mayor incidencia de disfunción cerebral mínima y defectos del lenguaje.

En resumen, el estado actual de los conocimientos no permite, por el momento predecir el crecimiento y desarrollo futuro del recién nacido pequeño para la edad gestacional. Parece que hay algunos factores maternos, fetales y perinatales que deprimen significativamente el desarrollo ulterior: magnitud del déficit del crecimiento $(2,10)$, gemelaridad (3), talla materna (10), peso de la placenta (17), anomalías del embarazo (81), situación socioeconómica $(2,10)$ y morbilidad perinatal.

\section{REFERENCIAS}

1.- Schutt, $W$. Foetal factors in intrauterine growth retardation. Clin. Develop. Med. 19; 1, 1965.

2.-Mc Donald, A. Retarded foela1 growth. Clin. Develop. Med. 19: [4, 1965.

3.-Warkany, J. Monroe B. and Sutherland, B. Intrauterine growth of retardation. Amer. J. Dis Child. 102: $127,1961$.

4.-Walker, J. Small for dates. Clinical Aspects. Proc. R. Soc. Med. 60: 877, 1967.

5.-Thompson, A, Billewicz, $W$, and Hytten, $F$. The assessment of fetal growth J. Obstet. Gynaec. Brit. Growth. 75: 903, 1968.

6.-Dawkins, $M$. The small for dates baby. Clin. Develop. Med. 19: 33, 1965.

7.-Räitü, C. Pregnancy, toxnemia and maternal cardiovascular efficiency. Clin. Develop. Med. 19: 50, 1965. 
8. Wigglesworth, J. Foetal Grrowth retardation. Brit. Med. Bull. 22: 13, 1966.

9.-- North, F. Small for dates neonates. I Maternal, gestational and neonatal characteristics. Pediatrics 38 : J013, 1966.

10.-Beargir, R., James, V, y Greene, J. Crecimiento y desarrollo de neonatos pequeños para la edad de gestación. Clín. Pediát. N. A. Febrero 159, 1970.

11.-Usher, $R$. Clinical and therapeutic aspects of fetal malnutrition. Pediat. Clin. N. A. 17; 169, 1970.

12.- Lichty, J. Ting, R. Bruns, P. and Dyar, E. Studies of babies born at bigh altitude $I$ relation of altitude to birth weight. Amer. J. Dis. Child. 93: 666, 1957.

13.-Walker, $C$. The infant of low birth weight. The practitioner 197: $281,1966$.

14.-Mc. Cunce, R. Food, growh and time. Lancet ?: 621, 1962.

15.-Wigglesworth, 1. Experionental growth retardation in the foetal rat. J. Path. Bact. 88: 1, 1964.

16.- Emmanowilides, G., Duane, G. Towsend, E. Dand, $J$. and Bauer, $R$. Effects of single umbilical artery ligation in the lamb fetus Pediatrics, 42: 919, 1968.

17,- Falkner, F. Prenatal influences upon small for da. tes infants, Padjal. Clinic. N. A. 17: 5, 1970.

17a.-Mc. Keown, T. and Record, R. Influence of placental size on foetal growth in man, with special reference to multiple pregancy. I. Endocrinol.. 9: 418,1963 .

18.- Hendricks, C. Patterns of fetal and placental growtb. Obst. Gynec. 24: 357, 1964.

19.-Winick, M. Coscia, A. and Noble A. Cellular growth in human placenta. I Normal placental growth. Pediatrics 39: 248, 1967.

20.-Filer, L. and Falkner, F. Perinatal influences on growth. Report from a twin study. XII Congreso Internacional de Pediatría, México, 1968.

21.- Thompson, A. In Hytten, F., Le, E. and Leitch, 1 . The physiology of human pregnancy Oxford, Blackvele Scientific Publications, 1964.

22.-Winick, M. Cellular growth of buman placenta II Intrauterine growth failure J. Pediat. 71: 390, 1967.

23.- Ting-Chao, W. and Latour, J. Microscopic measurement of the placental components in an attempt to assess the malnourished newborn infant. Am. J. Obst. Gynec. 94: 942, 1966.
24.- Shanklin, D. The iafluence of placental lesions on the newbom infant. Pediat. Clín. N. A. 17: 25,1970 .

25.-Dobbing, J. Vulnerable periods in developing brain. Applied Neuro-Chamistry. Oxford Blacwell, 1968.

26.--Gruenwald, P., Funakan'a, H. Mitani, S. Nishimura, $T$. and Talsenchi, $S$. Influence of enviromental factors on foetal growth in man. Lancet 1: 1026, 1967.

37.- Smilh, $C$. Effect of naternal undermutrition upon the newborn infant in Holland (194t-45). J. Pediat. 30: 229, 1947.

28.-Patirk, C. Nutritional adaptation to low dietary intakes of calories, proteins, vitamins and minerals in the tropics. Am. J. Clin. Nutr. 6: 1515, 1958.

29.-Mc Ganity, W., Cannon, R., Bridgforth, E. Aartin, M. Densen, P.. Newbill, J. Mc. Clean, S., Christie, $A$, Peterson, $C$. and Derby, $W$. The Vanderbilt cooperative study of maternal and infant nutri(ion. Am. J. Obstet. Gynecol. 67: 501, 1954,

30.- lyengar, L. Effects of dietary supplement on birth weight of infants. First Asian Congress of Nutrition. Hyderabad, India, 1971.

31.--Lechtig, A., Habieht, J. Leun E. y Guzmain, G. Influencia materna sobre el crecimiento fetal en poblaciones rurales de Guatemala, Arch. Latinoamer, de Nut. 22: I18, 1972.

32.-Ebbs, $S$, Tindall, $F$, and Ston, $\boldsymbol{W}$, The influence of prenatal diet on the mother and child, J. Clin, Nutr. 22: 515, 1941.

33,- Gruenwald, P. Some aspects of fostal distress. Clin. Develop. Med. 19: 66, 1965.

34.- Jerushalmy, J. Biostatisticial methods in investigations of child health Amer. I. Dis. Child. 114: $470,1967$.

35.-Scotr, $\boldsymbol{K}$. and Usher, $\boldsymbol{R}$. Fetal malnutrition. Its inci. dence, causes and effects. Amer. J. Obstet. Gynec. 94: $951,1966$.

36.- Buthler, N. Perinatal death Clin. Develop. Med. 19: 74,1965 .

37.-Bantaglia, F. and Lubchenco, L. A practical classification of newborn infants by weight and gestacional age. J. Pediat. 71: 159, 1967.

38.- Jerushalmy, J. von den Bers, B. Erhardt, $C$, and Jacobziner, H. Birth weight and gestation as indices of "immaturity" Amer. J. Dis. Child. 109: 43, 1965. 
39.- Girkentwald, P. Chronic fetiil distress and platental insufficiency. Biol. Neonatal. 5: 215, 1963.

40.-Beischer, N., Bhargava, V. Brown, J. and Smith, $M$. The incidence and significance of low oestriol excretion in an obstetric population. I. Obstet. Gynec. Cowlth. 75; 1024, 1968.

41.- Rizzardini, M., Duffau, G. Conireras, J. $\forall M e-$ neghello, J. Estudios en malnutrición fetal I. Examen físico y neurológico del recién nacido para estimar edad gestacional. Pediatría (Santiago) 12: 97. 1969.

42.-Amiel-Tizon, C. Neurological evaluation of the maturity of nowborn infants. Arch. Dis. Child. 43: 89. 1968.

43.-Minkawsky, A. and Sainte Ann-Dargassies, S. Physiopathologie foelale et accidents neurologiques an cours de la post maturité. Etud. Neonatal. 3: 225 , 1954.

44.-Dreyjus-Brisac, C. Flescher, J. and Plassart. E. L'electro encephalogramme: Critere d'age conceptionnel du neuveau-né a terme et premature Biol. Neonatal 4: 154, 1962.

45.-Michaelis, R., Schulte, F. and Nolte R. Motor behavior of small for gestational age newborn infants. J. Pediat. 76: 208, 1970.

46.-Farr, $V$. and Mitchell, $R$. The effect of birthweight on maturity scoring develop. Med. Child. Neurol. 9: $745,1967$.

47.-Usher, $R$. and Mc Lean, F. Intrauterine growth of live born. Caucasian infants at sea level. J. Pediat. 74: 901, 1969.

47a.-Lubchenco, I. Hausman, C., Dressier, M. and Boyd, $E$. Intrauterine growth as estimated from liveborn birth weight data at 24 to 42 weeks of gestation. Pediatrics 32: 793, 1963.

48.-Chifford, $S$. Postmaturity. Advances in Pediatrics 9: 13,1957 .

49.-Winick, M. Fetal malnutrition and future development. Pediatric Annals 2: 10, 1973.

50.-Cornblath, M., Wykegt, S., Baens, G. and Kleit, $\boldsymbol{R}$. Symptomatic neonatal hypoglycemai. Studies of carbohydrate metabolism in newborn infant VIII Pediatrics 33; 388, 1964.

51.-Las Heras, J., Dazzarola, P. y Rizzardini, M. Esludios en malnutrición fetal. II Glicemia y bilirnbinemia en el recién nacido a término pequeño para la edad gestacional. Rev. Chile. Pediat. 42: 255. 1971.
52.-Shelley, H. and Neligan, G. Neonatal Hypoglice. mia. Brit. Med. Bull. 22: 34, 1966.

53.- Melichar, V., Drahota, 2. and Hahn, P. Ketone bodies in the blood of full term newborn premature and dysmature infants of diabetic mothers Biol. Nconat. 11: 23, 1967.

54.-Melichar, $\boldsymbol{V}$. and Wolf, $\boldsymbol{H}$. Glycerin un freie fettsauren im blutplasma bei hypotrophen nengeboreกen. K]in. Wschr. 46: $549,1968$.

55.-Rubaliell, P., Formentin, P. and Tat's, L. Ammonia nitrogen, urea and uric acid blood levels in normal and hypodystrophic newborn. Biol. Neonat. 15: $129,1970$.

56.-Michaelis, R. and Melichar, $\boldsymbol{V}$. Serum bilitrubin in premature and hypotrophis newborns. Biol. Neonat. 12: 358,1968 .

57.-Bhaboa, $O$. Minimal rates of oxigen consumption in small for dates newborns babies. Arch. Dis. Childh. 45: 712, 1970.

58.-Sinclair, J. and Silverman, W. Relative hypermetabolism in undergrown human neonates. Lancet 2: 49.1964 .

59.- Sinclair, 1. and Sitverman. Intraulerine growth in active tissue mass of the human fetus, with particular reference to the under grown baby. Pediatrics 28: 48, 1966.

60.-Scopes, J. Metabolic rate and temperature control in human body. Brit. M. Bull. 22: 88, 1966.

61.- Sinclair, J. Heat production and thermoregulation in the small for date infant.. Pediat. Clin. N. A. 17: 147,1970 .

62.-Wigglesworth, J. Pathological and experimental aspects of foetal growth retardation. Pros. R. Soc. Med. 60: 879, 1967.

63.-Cassady, G. Body composition in intrauterine growth retardation. Pediat. Clin. N. A. 17: 79 , 1970.

64.- Kerpet-Fronius, E. Volume and composition of the body fluid compartments in severe infantile malnutrition. J. Pediat. 56: 826, 1960.

65.-Winick, $M$. Sellular changes during malnutrition. J. Pediat. 69: 987, 1966.

66.-Hubert, J. Abetson, H., Hathaway, W. Polycythemia in small for gestacional age infants. J. Pediat. 75: 812, 1969.

67.-Oski, F. and Naiman, J. Hematologic problems in the newborn Philadelphia W. B. Saunders, 1972. 
68.- Wells, $R$. Syndromes of hyperviscosity. New Engl. J. Med. 283: 183, 1970.

69.-Woot, $J$. Plethora in the newborn infant associated with cyanosis and convulsions: a review of post natal erythropotesis. J. Pediat. 54: 143, 1959.

70.-Bram, M. Valenzuela, J. y Rizzardini, M. Sindrome de hiperviscosidad sanguínea en el período neonatal. Pediatría 16, 1973.

71.- Kontras, S. Polycythermia and hiperviscosity syndromes in infants and children. Pediat. Clin. $N$. A. 19: 919,1972 .

72.-Scott, $\boldsymbol{K}$, and Usher, $\boldsymbol{R}$. Epiphyseal development in fetal malnutrition syndrome. New Engl. I. Med. 270: 822, 1967.

73.- Gruenwald, P. and Mink, H. Evaluation of body organ weights in perinatal pathology. Am. J. Clin. Patb. 34: 247, 1960.

74.-Naeye, R. Malnutrition: probable cause of fetal growth retardation. Arch. Path. 79: 284, 1965.

75.-Larroche, J. Maturation cérébrale et hypideveloppement pondéral du noeuvètu né. J. Neurological Sciencies 5; 39, 1967.

76.-Alvarado, S. Valenzuela, J. Schlager, G. Ferreiro, M. $y$ Rizzardini, $M$. Mortalidad y morbilidad del recién nacido hospitalizado según peso, edad gestacional y grado de crecimiento intrauterino, Rev. Chile, Pediat. 43: 9, 1972.

77.-Hurtado, Rizzardini, M. y Brown E. Cuantificación de inmuno globulina $G$ y de uno de los componentes del sistema complemento (C3) en recién nacidos a término normales $y$ con déficit de crecimiento intrauterino. Bol. Med. Hosp. Infant. (México) 29: 1, 1972.

78.- $A d d y, D$, Cord serum IgG levels in "small for dates" babies! Arch. Dis. Childh. 45: 809, 1970.

79.-Rantakallio, $P$. Groups at risk in low birth weight infants and perinatal mortality. Acta Paediat. Scand. Suply. 193. 1969.

80,-Battaglia, F. Frazier, T. and Hellegers, A, Bitth weight gestational age and pregnancy outcome, with specin] reference to hig birth weight-low-gestational age infunt. Pediatrics. 37: 417, 1966.
81.- Wolker, 1. Clinical obstetric features of prematurity and intrauterine growth retardation. Clin. Develop. Med. 19: 36, 1965.

81a.-Buttler, N. and Bonham, D. Perinatal mortality. Livingstone. Edinburgh, 1963.

82.- Ehrhardt, $C$. Influence of weight and gestacional on perinatal and neonatal mortality. Am J. Publ. Health 54: 1841, 1964.

83.-Oshi, P. and Naiman, J. Hematologic problems in the newborn Philadelphia 1972. W. B. Saunders Co. pp. $78-82$.

84.-Rizzardini, M. Dazzarola, P. y Las Heras, J. E\$tudios en malnutrición fetal III Crecimiento inmedialo y a largo plazo del recién nacido a término, pequeño para la edad gestacional. Bot. Méd. Hosp. Inf. (México) 29: 689, 1972.

85.-Babson, B. Growth of low birth weight infants. J. Pediat. 77: 11, 1970.

86.-Engleson, G. Rooth, G. and Tormblow, M. Followups study of dysmature infants. Arch. Dis Child. 38: 62, 1963.

87.-Fitzhardinge, $P$. and Steve, $E$. The small for date infant I Later growth patterna. Pediatrics 49: 671, 1972.

87a.-Drilien, $C$. The small for dates infants! Etiology and prognosis. Pediat. Clinic. N. A. 17: 9, 1970.

88,- Dawn, $M$. Levine, $M$. and New, $E$. A long term follow up study of small premature infants. Pediatrics 33: 945, 1964.

89.-Pick, W. The malnourished newborn. Pediatrics 33: $1.000,1964$.

90.-Babsan, S. and Kauges G. Preshool intelligence of undersized term infants. Amer. J. Dis. Child. 117: $553,1969$.

91.-Neligen, G. The clinical effects of being "Light for dates: Proc. R. Soc. Med. 60: 881, 1967.

92.-Fitzhordinge, $P$. and Steven, $E$. The small-for-date infant $I I$ Neurological and intellectual sequelae. Pediatrics 50: 50, 1972. 